\title{
INOVAÇÃO NO SETOR PÚBLICO: ANÁLISE DE UM ÓRGÃo ESTADUAL DE MEIO AMBIENTE E ESTUDO DE CASOS
}

\section{INNOVATION IN THE PUBLIC SECTOR: ANALYSIS OF A STATE ENVIRONMENTAL AGENCY AND CASE STUDIES}

\author{
E. S. FERNANDES ${ }^{1, *}$, F. A. D. VECCHIA ${ }^{1}$, R. VOLQUIND ${ }^{1,2}$ and B. D. GAYER ${ }^{1}$ \\ ${ }^{1}$ Federal University of Rio Grande do Sul, Department of Industrial Engineering, Porto Alegre, RS, Brazil \\ ${ }^{2}$ Fundação Estadual de Proteção Ambiental, Rio Grande do Sul, Brasil
}

${ }^{*}$ Corresponding author. Federal University of Rio Grande do Sul, Department of Industrial Engineering, Porto Alegre, RS, Brazil, Phone: +55 51 981002994 e-mail addressl: edu.silva.fernandes@gmail.com (E.S. Fernandes).

\begin{tabular}{l} 
A R T I C L E I N F O \\
\hline Article history: \\
Received 2020-01-24 \\
Accepted 2020-02-20 \\
Available online 2020-02-20 \\
pa l a vra - ch a ve \\
Inovação no setor público \\
Capacidade abortiva \\
Cultura inovativa \\
$k \begin{array}{l}k \text { y } w \text { o } r d s \\
\text { Public sector innovation } \\
\text { Absorptive capacity } \\
\text { Innovative culture }\end{array}$ \\
\hline
\end{tabular}

A B S T R A C T

Innovation has become increasingly common in the public sector, from small procedures to the adoption and installation of complex information technology systems. This study sought to analyze the public environmental agency characteristics in relation to innovation, such as innovation system, innovative culture, absorptive capacity, and appropriability. The research analyzed two case studies of the organization. The results showed that the agency does not have a formalized innovation system, but it has an innovative culture derived from both top management and operational area. It was also concluded that the absorptive capacity is the main factor explored in the search for improvements through innovation.

R E S U M O

A inovação tem se tornado cada vez mais comum no setor público, desde pequenas adaptações de procedimentos, até adoção e instalação de complexos sistemas de tecnologia da informação. Este estudo buscou analisar as características de um órgão público de meio ambiente em relação a temas inerentes à inovação, como sistema de inovação, cultura inovativa, capacidade absortiva e apropriabilidade. A pesquisa analisou dois estudos de caso da organização. Os resultados encontrados apontaram que o órgão não possui sistema de inovação formalizado, mas dispõe de cultura inovativa oriunda tanto da alta gestão como da área operacional. Também se concluiu que a capacidade absortiva é o principal fator explorado na busca por melhorias através da inovação. 


\section{INTRODUÇ̃̃̃O}

Inovar no setor público tem sido uma realidade crescente. Inovações tecnológicas, como adoção de programas e sistemas de computação para operacionalização dos seus cotidianos, registram maiores evidências. No entanto, a inovação não se limita apenas neste aspecto, estando relacionada a melhorias no dia-a-dia das organizações e de seus servidores, de forma estratégica (ARUNDEL \& HUBER, 2013).

A dinâmica da administração pública, caracterizada sobretudo pela grande rotatividade de gestores da alta direção, dificulta o aprimoramento de procedimentos, inclusive a adoção de padronizações (MOORE, 2010), especialmente quando tais instituições têm pouca autonomia nas tomadas de decisão. Os países latino-americanos historicamente viveram sob comportamento paternalista, cujo perfil era refletido na administração pública, interferindo na sua capacidade de prover serviços de qualidade. A partir da década de 1990, o setor público passou a receber demanda por modernização (CAVALCANTE \& CAMÕES, 2017), o que o levou a buscar alternativas para obtenção de eficiência, eficácia e efetividade na execução de suas atribuições.

A inovação mostrou-se oportuna para atender as demandas de qualidade, além de permitir que os gestores conquistem resultados, mostrando à sociedade sua capacidade de gestão e trazendo a estas as melhorias necessárias (CAVALCANTE \& CAMÕES, 2017). Seja uma iniciativa da própria gestão, muitas vezes imposta pelas diretorias, ou oriunda do corpo funcional, a inovação traz resultados positivos às organizações públicas e privadas (BORINS, 2001).

A partir do início dos anos 2000, ocorreu um aumento no número de licenças ambientais emitidas no estado do Rio Grande do Sul, como mostra a Figura 1. Este fato gerou a necessidade de melhorias da organização responsável pela emissão destas licenças, que é caracterizada por problemas comuns do setor público ambiental, como recursos humanos sem desenvolvimento de todas habilidades necessárias e em quantidades reduzidas, bem como dificuldades financeiras (ULIBARRI, CAIN, \& AJAMI, 2017). No caso específico do estudo, os dirigentes, frente às dificuldades apontadas acima, entenderam que uma forma de suprir carências é modernizar a instituição de forma a poder realocar os recursos existentes, reduzindo a necessidade de contratação de novos servidores ou aquisição de equipamentos estruturais. Sendo assim, este artigo tem como objetivo avaliar o potencial de inovação de um órgão público estadual do Rio Grande do Sul.

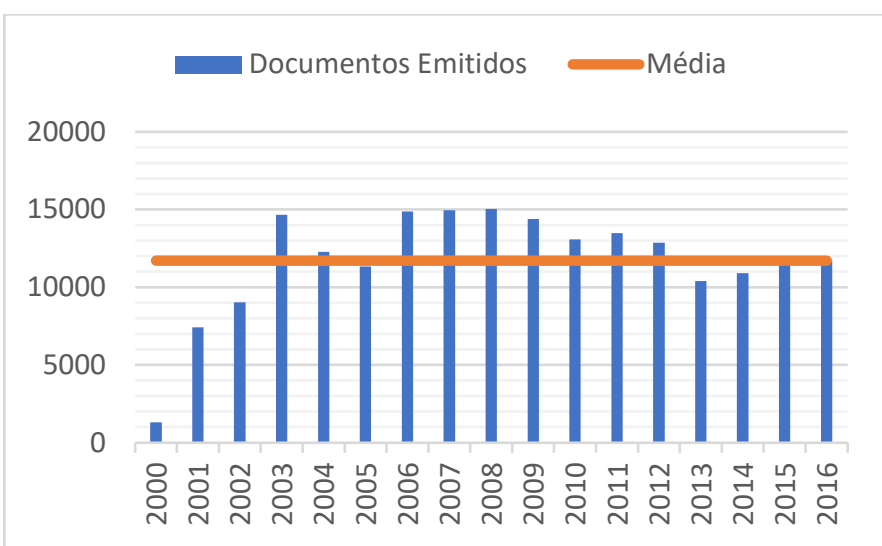

Figura 1 - Evolução na quantidade de licenças ambientais emitidas pela FEPAM. Fonte: fepam.rs.gov.br

A partir do conhecimento do que está sendo discutido no mundo atual sobre inovação no setor público, foi efetuada uma avaliação sobre o comportamento do órgão específico a respeito de como são criadas e adotadas as inovações no seu dia-a-dia, associando-as a conceitos do planejamento e organização institucional, verificando o quanto a cultura organizacional é adepta a novas descobertas e adoções de procedimentos. Também buscou-se associar conceitos teóricos da área de inovação, tais como cultura inovativa, capacidade inovativa, sistema de inovação, apropriabilidade, adoção e inovação disruptiva, de modo a qualificar a instituição no meio inovativo. Por fim, uma avaliação sobre dois estudos de caso ilustra a experiência da organização como adepta de inovações no seu cotidiano, onde busca melhorias dentro de um quadro de necessidade de avanços e poucos recursos disponíveis, humanos, estruturais e financeiros.

Nas seções seguintes são apresentados o referencial teórico do tema inovação no setor público, a metodologia do presente estudo, os resultados obtidos e, por fim, conclusões e propostas de novas pesquisas que podem dar continuidade ao tópico.

\section{REFERENCIAL TEÓRICO}

Ao se evoluir os processos, o foco das empresas vem migrando da eficiência para a competitividade, com o aumento da importância da qualidade, e mais recentemente com o desafio da inovação (LIVRAMENTO, OLIVEIRA, \& MORAES, 2015). Ou seja, a inovação tem se mostrado parte do objetivo de muitas organizações. Além de ser um dos esforços comumente associados ao desempenho econômico e social de empresas, governos e regiões (GOEDHUYS e VEUGELERS, 2012).

O conceito de sistemas de inovação foi desenvolvido para analisar os subsistemas sociais e atores que contribuem de algum modo, intencionalmente ou não, seja de forma direta ou indireta para a inovação (HEKKERT et al, 2007). É uma interrelação de atores e instituições em uma região particular que permite geração, difusão e apropriação de inovação (CHUNG, 2002). 
Segundo diversos estudos, um sistema de inovação pode ser avaliado por meio dos seguintes fatores-chave: (i) região, onde se busca alguma homogeneidade nos aspectos sociais e culturais, (ii) inovação em si, onde se avalia os esforços inovativos em produtos, processos e organizacionais, (iii) rede e interação, que diz respeito ao nível de interação e cooperação entre os atores envolvidos e à comunicação formal e informal para troca de conhecimento interno e externo à organização e finalmente, (iv) aprendizagem, onde se avalia o nível de capacidade absortiva e de desenvolvimento (COOKE, 2001).

\subsection{Cultura Inovativa}

Ao passo que as organizações percebem a mudança como inevitável, outros fatores, como valores, crenças e padrões de comportamento, começam a ganhar importância para as análises e meios que permeiam o desenvolvimento da cultura inovativa e, por consequência, o desempenho organizacional (MARTINS \& MARTINS, 2002). Assim como tais fatores promovem a cultura inovativa, esta, por sua vez se traduz em um ambiente de trabalho com maior dinamismo, incentiva a criatividade, encorajando a liberdade e iniciativa individual (ROCHA, PELOGIO, \& AÑEZ, 2013).

A partir do incentivo do comportamento inovativo, é possível desenvolver a capacidade inovativa. A capacidade de inovação é definida como as habilidades e os conhecimentos necessários para efetivamente absorver, dominar e melhorar as tecnologias existentes e criar novas (LALL, 1992).

\subsection{Capacidade Absortiva e Apropriabilidade}

A capacidade absortiva representa os esforços à capacidade de orientação ao mercado e à capacidade de assimilar novas tecnologias e conhecimentos para tornar possível a capacidade de inovar da organização (COHEN e LEVINTHAL, 1990). É fundamental para a apropriação de inovações externas e têm um efeito positivo na busca de inovações incrementais (RITALA \& HURMELINNALAUKKANEN, 2009). Além disso, é comum que se busque novas tecnologias e maneiras mais eficazes de realizar os processos, assim, as melhores tecnologias tendem a ser adotadas por outras empresas (DOSI \& NELSON, 1994).

Segundo Cohen e Levinthal (1989), as características do conhecimento referentes à facilidade de aprendizado são determinantes e influenciam diretamente em como a organização identifica oportunidades de absorção e apropriabilidade de novas tecnologias e inovações, pois há condições de identificar e avaliar a utilidade e qualidade de tecnologias externas.

\section{METODOLOGIA}

Este trabalho pode ser classificado de acordo com a abordagem como sendo qualitativo. Já segundo os objetivos pode ser caracterizado como sendo uma pesquisa descritiva. Além disso, de acordo com os procedimentos técnicos utilizados este trabalho pode ser classificado como um estudo de caso, que tratam da implantação de dois processos realizados pela FEPAM para automatizar e otimizar o processo de licenciamento: a renovação automática de licenças e o Sistema Online de Licenciamento - SOL. No caso específico da renovação automática de licenças foi analisada a Lei
Complementar No 140/2011 e a Portaria FEPAM No 46/2015.

A Fundação Estadual de Proteção Ambiental - FEPAM, é um órgão público ligado a administração indireta e vinculado à Secretaria Estadual do Ambiente e Desenvolvimento Sustentável - SEMA, responsável principalmente pela emissão de licenças ambientais no estado do Rio Grande do Sul. Possui um orçamento anual de 90 milhões de reais e recebe cerca de 12 mil pedidos de licenciamento ambiental por ano. Possui cerca de 300 servidores, aonde aproximadamente 175 trabalham diretamente com o licenciamento. Isto equivale a uma taxa mensal de 5,7 licenças por servidor. Atualmente, uma licença ambiental demora em média 114 dias para ser emitida.

Para a realização deste trabalho, foram realizadas entrevistas com membros da alta gestão da FEPAM, onde foram coletadas opiniões e informações a respeito da aplicação de conceitos como cultura e capacidade inovativa e capacidade absortiva na organização em questão. Os dados das Figuras 1 e 2 foram retirados diretamente do website da FEPAM.

\section{RESULTADOS E DISCUSSÕES}

A FEPAM se caracteriza por ser uma instituição onde a gestão institucional nunca foi formalizada. Apenas em 2015 houve a realização de um planejamento estratégico, empírico, com identificação de eixos de atuação e definição de objetivos. A partir destes, ao longo dos últimos três anos, foram estabelecidas metas vinculadas ao licenciamento ambiental.

A estrutura técnica não conta com um setor dedicado ao planejamento organizacional ou à busca por melhorias. Atualmente encontra-se em avaliação uma revisão estatutária onde será criado um setor de organização de métodos, que centralizará as informações pertinentes a procedimentos e padronizações vinculadas ao licenciamento, hoje dispersas pela instituição. Também não existe um setor que centralize, gerencie ou mesmo incentive inovações. Dificuldades quanto à disponibilidade financeira completam o panorama. Sem recursos financeiros, as inovações limitavam-se a pequenas ações, de baixo custo.

\subsection{A inovação dentro da instituição}

A instituição adota inovações pontuais, oriundas e estabelecidas dentro de setores, a partir de demandas do dia-adia. A resistência a mudanças por parte do corpo técnico inibe a difusão de novos métodos e ferramentas, dificultando sua permeabilidade junto a outros setores, ainda que executem atividade idêntica.

Qualquer inovação que tenha surgido no passado foi por iniciativa do corpo funcional, para melhorias pontuais nas suas ações cotidianas. Diversos foram os exemplos ao longo dos anos, como procedimentos e padrões adotados por setores distintos, sem difusão entre aqueles que poderiam fazer uso destas novidades. Um destaque que exemplifica bem a origem das iniciativas e a ausência da difusão foi o estabelecimento do atual sistema de licenciamento ambiental, que padronizou pareceres técnicos, os quais, por sua vez, geram as licenças ambientais.

A área de licenciamento de atividades industriais idealizou, desenvolveu e implantou o sistema, chamado de geração automática de pareceres. O conteúdo dos pareceres, inicialmente de renovações de Licenças de Operação, foi 
padronizado e vinculado ao tipo e ao tamanho da atividade industrial. Embora fosse facilmente replicável por outros departamentos, a geração automática de pareceres levou mais de dez anos para ser compreendida e difundida por toda a instituição, tendo sua aplicação concluída apenas em 2015. A difusão plena só foi possível, todavia, por determinação da diretoria, que impôs a todos os setores a adoção.

Verifica-se, portanto, que há capacidade inovativa dentro da instituição e que esta é exercida basicamente pela área operacional. A busca por soluções de diversos problemas sem recursos financeiros é comum e utiliza de muita criatividade, a qual tem sido incentivada, principalmente conduzida através da mudança de métodos de produção.

Algumas características inovativas se sobressaem, devido a condições impostas pelo meio. Assim, adoção de ações e ferramentas desenvolvidas por outras instituições do mesmo ramo de atuação no país representam o tipo de inovação mais utilizado. Facilmente compreendidas e obtidas junto aos pares nacionais, as novidades são rapidamente adaptadas à realidade institucional do órgão gaúcho.

Com a finalidade de ilustrar as características observadas, duas experiências da FEPAM foram avaliadas, identificando-se como a instituição se comporta perante o tema da inovação. A primeira inovação foi uma alteração de procedimento com base em uma situação peculiar ocasionada pela legislação brasileira, em especial pela Lei Complementar $\mathrm{N}^{\mathrm{o}} 140 / 2011$, onde licenças ambientais eram prorrogadas por prazo indeterminado a partir do pedido de sua renovação, desde que a solicitação fosse efetuada em prazo superior a 120 dias de seu vencimento. O segundo caso avaliado é a adoção de tecnologia de informação através de um sistema informatizado para gerenciamento dos pedidos de licenciamento e requerimento das licenças ambientais.

\subsection{Renovação automática de licenças ambientais - Portaria $N^{0}$ 46/2015}

O Conselho Nacional do Meio Ambiente - CONAMA - publicou em 1997 a Resolução No 237, trazendo em seu artigo 18 , inciso I, parágrafo $4^{\circ}$ :

A renovação da Licença de Operação (LO) de uma atividade ou empreendimento deverá ser requerida com antecedência mínima de 120 (cento e vinte) dias da expiração de seu prazo de validade, fixado na respectiva licença, ficando este automaticamente prorrogado até a manifestação definitiva do órgão ambiental competente (Brasil, RESOLUÇÃO CONAMA n ${ }^{\circ} 237$, de 19 de dez. de 1997. , 1997).

Tal determinação foi revista e atualizada em 2011 pela Lei Complementar $N^{\circ} 140$, através do artigo 14, parágrafo $4^{\circ}$, ampliando-a para todos os tipos de licença ambiental:

A renovação de licenças ambientais deve ser requerida com antecedência mínima de 120 (cento e vinte) dias da expiração de seu prazo de validade, fixado na respectiva licença, ficando este automaticamente prorrogado até a manifestação definitiva do órgão ambiental competente (Brasil, Lei Complementar 140, de 08 de dez. de 2011, 2011).

Portanto, uma vez solicitada renovação de qualquer licença ambiental com a antecedência exigida e não havendo conclusão e manifestação por parte do órgão ambiental em relação ao novo pedido, a licença original era prorrogada por prazo indeterminado, até que ocorresse manifestação quanto ao novo pedido.

A FEPAM compreendeu que a situação de prorrogação de uma licença, sem o devido acompanhamento de seu atendimento, com fiscalização deficitária quanto ao seu cumprimento e, muitas vezes, devido à falta de solução, colabora para formação de grandes estoques de pedidos de licenciamento. O hábito de efetuar ação de vistoria in loco ao empreendimento por conta da concessão de renovação de uma licença fazia com que o processo demandasse muito tempo para que uma licença fosse renovada. Além disso, a receita financeira do órgão ambiental fica comprometida, uma vez que o período de prorrogação faz com que o requerente da renovação da licença seja beneficiado pelo fato de o tempo da licença vigente ser postergado, adiando-se também a data de um novo pagamento para obtenção do documento correspondente ao novo ciclo.

Desta forma, as lideranças do corpo técnico de analistas responsáveis pelo licenciamento ambiental trouxeram para a direção à proposta de renovar automaticamente as licenças ambientais solicitadas nos últimos quatro anos - prazo de vigência de uma Licença de Operação à época (Rio Grande do Sul, Resolução CONSEMA N ${ }^{\circ}$ 038/2003, de 18 de jul. de 2003, 2003). Através de uma programação, o sistema existente de concessão de licenças copiaria a última licença emitida do empreendimento, concedendo-lhe novos 4 anos de vigência, sem responsabilizar os analistas da instituição. A renovação contaria com regras de requisitos, relacionados a: solicitação de renovação efetuada dentro do período de vigência do documento original, licença original do empreendimento não ser objeto de suspensão administrativa ou judicial e pagamento integral do ressarcimento dos custos para análise de pedido de licenciamento.

A partir da efetivação da nova proposta, concretizada pela Portaria No 46 (Rio Grande do Sul, Portaria FEPAM 46, de 07 de mai. de 2015, 2015), bons resultados passaram a ser obtidos. Imediatamente no mês de publicação da Portaria, mais de mil licenças foram emitidas, reduzindo significativamente o estoque de processos por avaliar. Destas, mais de $80 \%$ encontravam-se na antiga situação de prorrogação. No ano de publicação foram emitidos mais de 1500 documentos de forma automática. No ano seguinte, este número foi reduzido para cerca de 800 , enquanto no terceiro ano apenas 483 licenças sofreram renovação automática. A Figura 1 mostra claramente a redução do estoque causada pela adoção da nova metodologia no mês de seu início. 


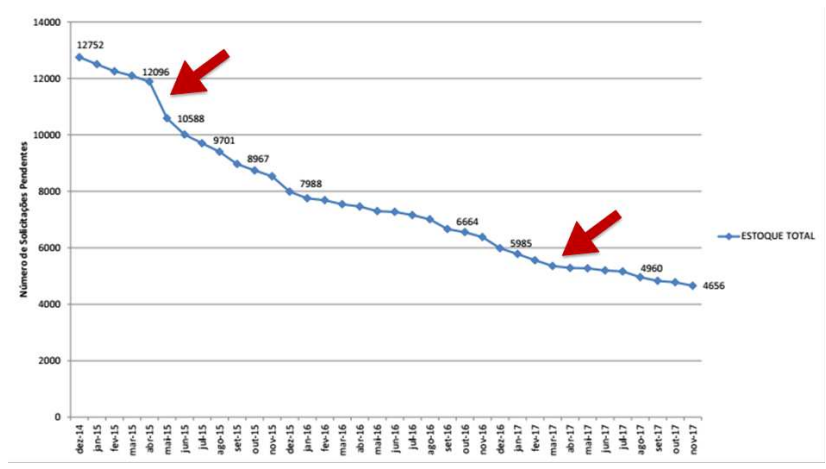

Figura 2- Evolução no estoque de solicitações de licenças ambientais. Fonte: fepam.rs.gov.br

Associado à renovação automática, a FEPAM deveria estabelecer operações de fiscalização aos empreendimentos beneficiados pela nova regra, verificando sua eficácia em termos de cumprimento às condicionantes das licenças renovadas automaticamente. Tal fiscalização seria efetuada em amostras aleatórias. Em recente operação de fiscalização realizada no ano de 2017, apenas $30 \%$ dos empreendimentos vistoriados apresentaram alguma inconformidade com a licença ambiental emitida de forma automática.

Assim, verificou-se que a inovação trazida pela área operacional trouxe benefícios diretos ao processo de licenciamento ambiental. A sensível redução do estoque no ano inicial, seguida por quantidades menores de documentos emitidos nos anos subsequentes, caracterizou a medida como de relevância temporária, mas fundamental para a redução dos estoques acumulados. $\mathrm{O}$ fato de a grande maioria das licenças renovadas automaticamente terem sido dadas a empreendimentos cuja licença estava prorrogada demonstrou que não houve prejuízo técnico-ambiental pela medida adotada. A certificação final de bons resultados foi trazida pelas operações de fiscalização, que encontraram percentual reduzido de empreendimentos em situação de inconformidade com as licenças concedidas de forma automática.

\subsection{Sistema Online de Licenciamento - SOL}

A demanda moderna por velocidade na prestação de serviços, por transparência e disponibilidade de informações em tempo real, por adoção de critérios objetivos e por sistematização de workflows levou o órgão ambiental a buscar inovação para atualizar-se. Até a implantação deste novo sistema, os pedidos eram analisados a partir de informações disponibilizadas em meio físico - papel - e não podiam ser avaliadas simultaneamente por mais de um analista, uma vez que o processo devia realizar, fisicamente, o caminho de análise.

A partir do conhecimento da alta direção de um sistema utilizado por outro órgão ambiental de nível estadual, a FEPAM optou por adotar o seu próprio, a partir de conceitos apresentados pelo equivalente do outro estado. Tal decisão por adaptação, ao invés da simples adoção fundamentou-se no fato de que o sistema desenvolvido pelo seu par apresentava excelente interface entre requerente e órgão ambiental, guiando-o por todo o caminho, até a conclusão do requerimento de licenciamento ambiental. No entanto, a FEPAM dispunha de sistema de emissão de pareceres técnicos automatizados, ao contrário do que foi verificado no outro órgão. Assim, foi desenvolvido o Sistema Online de Licenciamento (SOL), com três premissas fundamentais: (i) digitalização dos processos, deixando de recebê-los impressos e permitindo análises simultâneas; (ii) cadastramento de informações no sistema de processamento de pareceres técnicos; e (iii) possibilidade de consulta online de todas as informações por qualquer cidadão, concedendo a transparência necessária a um órgão público.

Além da adoção, verifica-se que o desenvolvimento do SOL representa a capacidade absortiva da instituição. Normalmente esta característica é associada a competição e concorrência entre empresas desenvolvedoras. Neste caso, no entanto, mesmo que não formalizada, a capacidade absortiva ficou representada pela aceitação do novo sistema, que foi oferecido à FEPAM pelo órgão parceiro. Também pode a adoção e o desenvolvimento do SOL serem considerados como uma apropriabilidade, uma vez que houve aprimoramento da ideia original, tornando-a mais atrativa e acessível.

O Sistema teve desenvolvimento a partir de junho de 2016 e foi lançado em fevereiro de 2017. As inovações foram concentradas nos conceitos de transparência, através do acesso de todos os documentos por qualquer cidadão e do envio de comunicados automáticos a cada movimentação do processo, e de agilidade e velocidade, por meio da análise simultânea das informações e ao fato de não serem criados processos sem todas as informações inseridas corretamente.

Como resultados, o SOL respondia em novembro de 2017 por $30 \%$ da composição do estoque pendente de solução, $45 \%$ de todos os processos de requerimento de licenciamento ambiental concluídos (deferidos, indeferidos ou arquivados), com tempo médio de atendimento de 80 dias. Confirmou-se assim que a adoção do Sistema, com absorção de tecnologia e adaptações, trouxe benefícios a curto prazo ao órgão ambiental.

\section{CONCLUSÃO}

Ao longo deste trabalho verificou-se que há bastante espaço para implantação de inovações na Fundação. No entanto, a característica informal do sistema de inovação, por não existirem incentivos à busca por novas tecnologias e procedimentos, mas aceitação, as inovações acabam não sendo implantadas de maneira sistemática, nem mesmo são difundidas dentro da instituição.

Verificou-se que a maior parte da inovação existente na organização é proveniente da área operacional, comprovando o trazido por Borins (2002), que as inovações no setor público costumam emanar da alta direção para situações de crise e das áreas operacionais para melhorias do dia-a-dia. Nos dois exemplos trazidos, a Renovação Automática, de caráter operacional, com influência direta no dia-a-dia, teve origem na área operacional, enquanto o SOL, uma demanda de porte maior, com maior impacto na Fundação e entre seus clientes, é oriunda da alta direção.

As inovações da instituição, mesmo dentro de um sistema informal e oriundas predominantemente dos funcionários, são exitosas nos objetivos de melhorias dos procedimentos. A Renovação Automática representou cerca de $10 \%$ dos processos solucionados desde sua implantação, colaborando para que a Fundação pudesse trabalhar em outras de suas atribuições. O SOL, por sua vez, que se encontra ainda em desenvolvimento, resultou em atualização de conceitos, como a abdicação do uso de processos impressos e a 
acessibilidade a qualquer momento, de qualquer lugar, essencial nos dias de hoje.

Sobretudo, no entanto, foi demonstrado que o setor público é adequado para implantação de inovações, tanto tecnológicas como procedimentais, garantindo melhores desempenhos na prestação de serviços à população. Muitas vezes estas inovações são implantadas sob baixo custo financeiro e com pequenas adaptações, garantindo aceitação por rápida por parte das altas gestões, devendo ser incentivadas em todas as instituições públicas

\section{R E F E R Ê N C I A S}

ARUNDEL, A.; HUBER, D. From too little to too much innovation? Issues in measuring innovation in the public sector. Structural Change and Economic Dynamics, v. 27, p. 146-159, 2013.

BORINS, S. Encouraging innovation in the public sector. Journal of Intellectual Capital, v. 2, n. 3, p. 310-319, 2001.

BORINS, S. Leadership and innovation in the public sector. Leadership \& Organization Development Journal, v. 23, n. 8, p. 467-476, 2002.

BRASIL. RESOLUÇÃO CONAMA n 237, de 19 de dez. de 1997. Regulamenta os aspectos de licenciamento ambiental estabelecidos na Política Nacional do Meio Ambiente., p. 30841-30843, 22 dez. 1997. Disponivel em:

<http://www.mma.gov.br/port/conama/res/res97/res237 97.html>. Acesso em: 01 jan. 2018.

BRASIL. Lei Complementar 140, de 08 de dez. de 2011. Fixa normas, nos termos dos incisos III, VI e VII do caput e do parágrafo único do art. 23 da Constituição Federal, dez 2011. Disponivel em: <http://www.planalto.gov.br/ccivil_03/leis/LCP/Lcp140 .htm>. Acesso em: 01 jan. 2018.

CAVAlCANTE, P.; CAMÕES, M. Do the Brazilian innovations in public management constitute a new model? RAI Revista de Administração e Inovação, v. 14, p. 90-96, 2017.

CHUNG, S. Building a national innovation system through regional innovation systems. Technovation, v. 22, p. 485-491, 2002.

COHEN; LEVINTHAL. Innovation and learning: the two faces of R \& D. The Economic Journal, v. 99, n. 397, p. $569-596,1989$.
DOSI, G.; NELSON, R. R. An introduction to evolutionary theories. Journal of Evolutionary Economics, v. 4, n. 3, p. 153-172, 1994.

LALL, S. Technological capabilities and industrialization. World Development, v. 20, n. 2, p. 165-186, 1992.

LIVRAMENTO, T. F. S.; OLIVEIRA, E. A. D. A. Q.; MORAES, M. B. D. Empresas resilientes: o desafio de estabelecer uma cultura inovativa como fator de proteção. Latin American Journal of Business Management, v. 6, n. 2, p. 237-255, 2015.

MARTINS, E.; MARTINS, N. An organizational culture model to promote creativity and innovation. Journal of Industrial Psychology, v. 28, n. 1, p. 58-65, 2002.

MOORE, M. H. Break-Through Innovations and Continuous Improvement: Two Different Models of Innovative Processes in the Public Sector. Public Money \& Management, v. 25, n. 1, p. 43-50, 2010.

RIO GRANDE DO SUL. Resolução CONSEMA N 038/2003, de 18 de jul. de 2003. Estabelece procedimentos, critérios técnicos e prazos para Licenciamento Ambiental realizado pela Fundação Estadual de Proteção Ambiental - FEPAM, no Estado do Rio Grande do Sul., jul 2003. Disponivel em: <http://www.sema.rs.gov.br/resolucoes>. Acesso em: 01 jan. 2018.

RIO GRANDE DO SUL. Portaria FEPAM 46, de 07 de mai. de 2015. Institui o procedimento para renovação automática de Licenças Ambientais e Certificados no âmbito de competências da Fundação Estadual de Proteção Ambiental., 07 mai 2015. Disponivel em: <http://www.sema.rs.gov.br/resolucoes>. Acesso em: 01 jan. 2018.

RITALA, P.; HURMELINNA-LAUKKANEN, P. What's in it for me? Creating and appropriating value in innovationrelated coopetition. Technovation, v. 29, n. 12, p. 819828, 2009.

ROCHA, L. C. S.; PELOGIO, E. A.; AÑEZ, M. E. M. Cultura e clima organizacionais: um estudo em indústrias de laticínios do Estado do Rio Grande do Norte. Gestão da Produção, v. 20, n. 2, p. 455-468, 2013.

ULIBARRI, N.; CAIN, B. E.; AJAMI, N. K. A Framework for Building Efficient Environmental Permitting Process. Sustainability, v. 9, n. 180, 2017. 